\title{
Peritoneal Histoplasmosis about a Case and Literature Review
}

\section{Brahim Moulaye El Hassen ${ }^{*}$, Noukhoum Koné2, Mohamed Salem Mouammar ${ }^{3}$, Moulay Ahmed Moulay Hachem ${ }^{4}$}

\author{
${ }^{1}$ National Oncology Center, Nouakchott, Mauritania \\ ${ }^{2}$ Department of Neurosurgery, Kiffa Hospital Center, Kiffa, Mauritania \\ ${ }^{3}$ Department of Visceral Surgery, Cheick Zayed Hospital, Nouakchott, Mauritania \\ ${ }^{4}$ National Police Medical Center, Nouakchott, Mauritania \\ Email: ^b.moulayelhassen@hotmail.com
}

How to cite this paper: El Hassen, B.M. Koné, N., Mouammar, M.S. and Hachem, M.A.M. (2021) Peritoneal Histoplasmosis about a Case and Literature Review. Health, 13, 1065-1070.

https://doi.org/10.4236/health.2021.131007 $\underline{9}$

Received: July 10, 2021

Accepted: October 9, 2021

Published: October 12, 2021

Copyright (c) 2021 by author(s) and Scientific Research Publishing Inc. This work is licensed under the Creative Commons Attribution International License (CC BY 4.0).

http://creativecommons.org/licenses/by/4.0/

\begin{abstract}
Histoplasmosis is an opportunistic granulomatous fungal infection. Peritoneal histoplasmosis $(\mathrm{PH})$ is a rare form. The first case of $\mathrm{PH}$ was described in 1970 but this is the first case reported in Mauritania. We report the case of a 60 -year-old male patient with a history of pulmonary tuberculosis, treated and declared cured, and partial epileptic seizures treated with Carbamazepine. Contrast computed tomography of the abdomen showed a large mass with a large intraperitoneal fluid component with a finely calcified wall in places, for which laparoscopy and biopsy were performed, identifying Histoplasma capsulatum infection. The subject received treatment with amphotericin $B$ deoxycholate with good evolution, and outpatient management with itraconazole. $\mathrm{PH}$ is a rare entity that requires high clinical suspicion, especially in immunocompetent patients. The patient was informed that non-identifying information from the case would be submitted for publication, and he provided consent.
\end{abstract}

\section{Keywords}

Peritoneal Histoplasmosis, Histoplasma capsulatum var. duboisii, Pathological Anatomy, Mauritania

\section{Introduction}

Histoplasmosis caused by Histoplasma capsulatum var. duboisii (H. duboisii) is a profound opportunistic mycosis, endemic in Africa. The precise epidemiology of H. duboisii infection and its pathogenesis remains poorly understood. Histoplasmosis has a wide range of clinical presentations that depend primarily on 
three factors: fungal load, virulence and Histoplasma strain, and host immune status [1].

More commonly involving organs such as the liver, spleen, bone marrow and skin [2]. Peritonitis associated with $H$. capsulatum is extremely rare, with few cases reported in the literature. Risk factors for developing fungal peritonitis include previous antibiotic use, immunosuppression status, environmental exposure, intra-abdominal surgery, and extraperitoneal spread of fungal infection [3]. We report the first observation in Mauritania of an isolated peritoneal localization of $H$. duboisii histoplasmosis in an immunocompetent patient.

\section{Patient and Observation}

The 60-year-old male patient had emigrated from Mauritania to Guinea Bissau, Congo and Senegal; he had a history of pulmonary tuberculosis, treated and declared cured, and partial epileptic seizures treated with carbamazepine.

The clinical examination noted abdominal pain giving the impression of heaviness. The remainder of the clinical examination, particularly pulmonary, neurological, lymph node and locomotor, was unremarkable. Abdominal computed tomography (CT) (Figure 1), sagittal section revealed a large mass with a large intraperitoneal fluid component with a finely calcified wall in places, located opposite the 3rd, 4th, 5th lumbar vertebrae, and plunging in the pelvis by its lower pole above the bladder, measuring $157 \times 100 \mathrm{~mm}$. Anatomopathological examination of the operative parts (Figure 2) revealed Histoplasma capsulatum var. duboisii stained with eosin hematoxylin at $\times 400$ magnification shows a rounded or oval, corresponding to a bulky nucleus surrounded by a pseudo capsule producing a clear halo appearance (Figure 3(a)). Histoplasmosis capsulatum var. duboisii at $\times 400$ magnification showing the yeast-like appearance

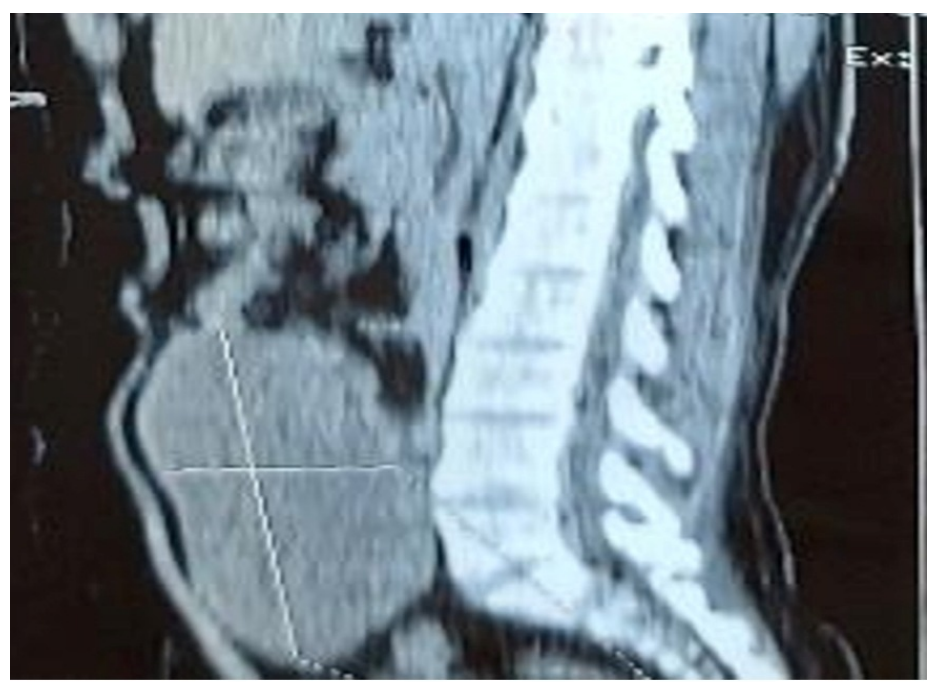

Figure 1. Abdominal CT scan sagittal section revealed a large mass with a large intraperitoneal fluid component with a finely calcified wall in places, located opposite the $3 \mathrm{rd}$, 4th, 5th lumbar vertebrae, and plunging in the pelvis by its lower pole above the bladder, measuring $157 \times 100 \mathrm{~mm}$. 

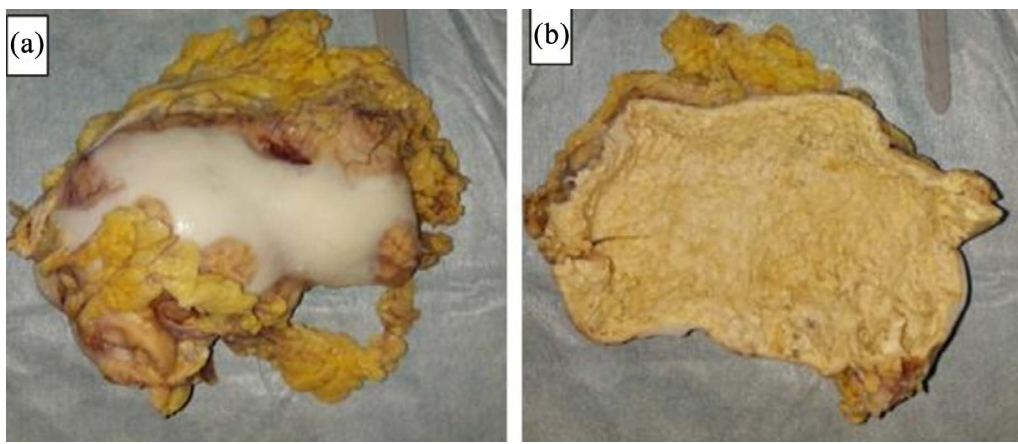

Figure 2. Macroscopic view of operating parts.
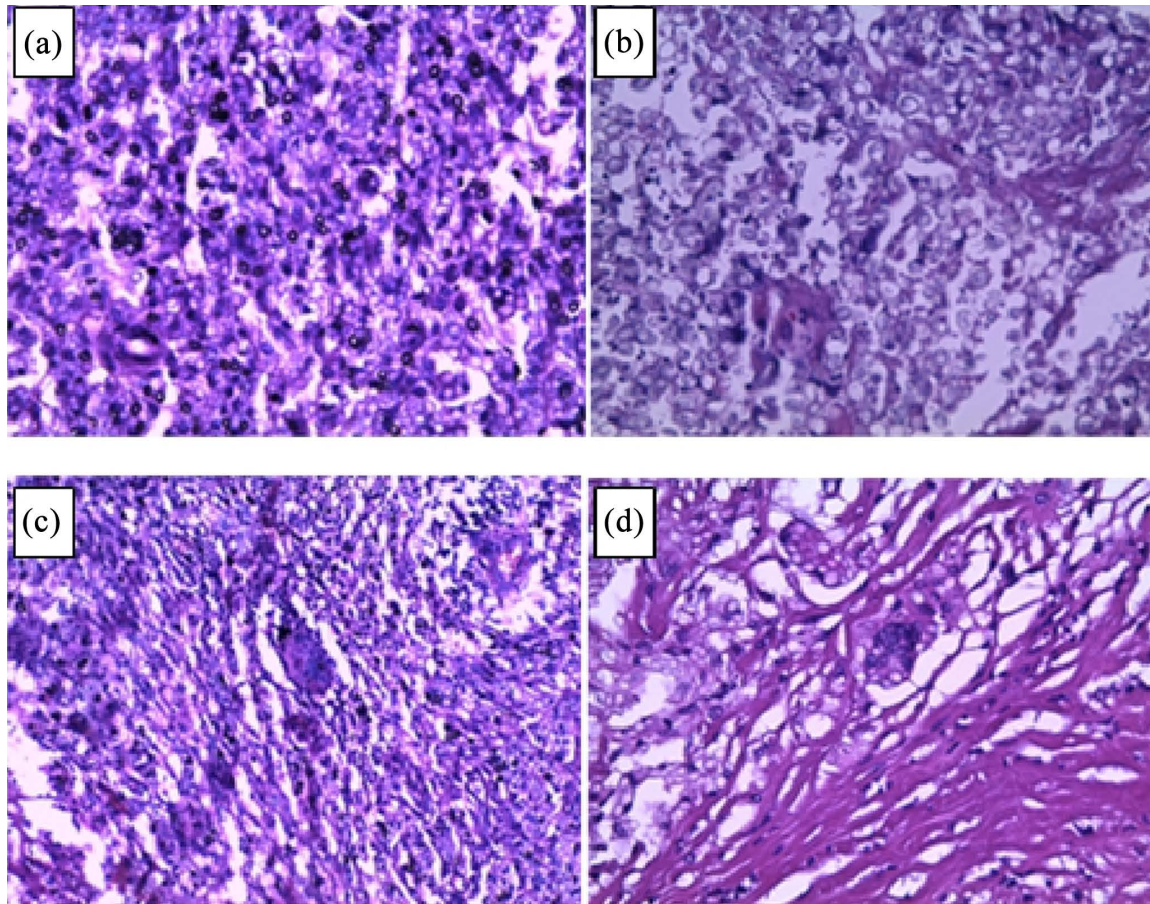

Figure 3. (a) Histoplasma capsulatum var. duboisii stained with eosin hematoxylin at $\times 400$ magnification shows rounded or oval, corresponding to a bulky nucleus surrounded by a pseudo capsule producing a clear halo appearance. (b) Histoplasmosis capsulatum var. duboisii at $\times 400$ magnification showing the yeast-like appearance with double contour. (c) Epitheloid and gigantocellular granuloma, without necrosis, stained with eosin hematoxylin at $100 \times$ magnification. (d) Multinucleated giant cells mixed with yeast-like aspects dissociating fibrous tissue stained with hematoxylin-eosin at $\times 400$ magnification.

with double contour (Figure 3(b)). Epitheloid and gigantocellular granuloma, without necrosis, stained with eosin hematoxylin at $100 \times$ magnification (Figure $3(\mathrm{c})$ ). Multinucleated giant cells mixed with yeast-like aspects dissociating fibrous tissue stained with hematoxylin-eosin at $\times 400$ magnification (Figure 3(d)). Staining by Periodic Acid Schiff (P.A.S) stains these forms purple-pink, with the pseudo-capsule visible (Figure 4(a), Figure 4(b)). Gomori-Grocott silver impregnation at high magnification $\times 400$ shows the wall stained in black of the yeasts in intrahistiocytic position dispersed in the necrosis (Figure 5(a)). And the black-colored wall of the yeasts (Figure 5(b)). 

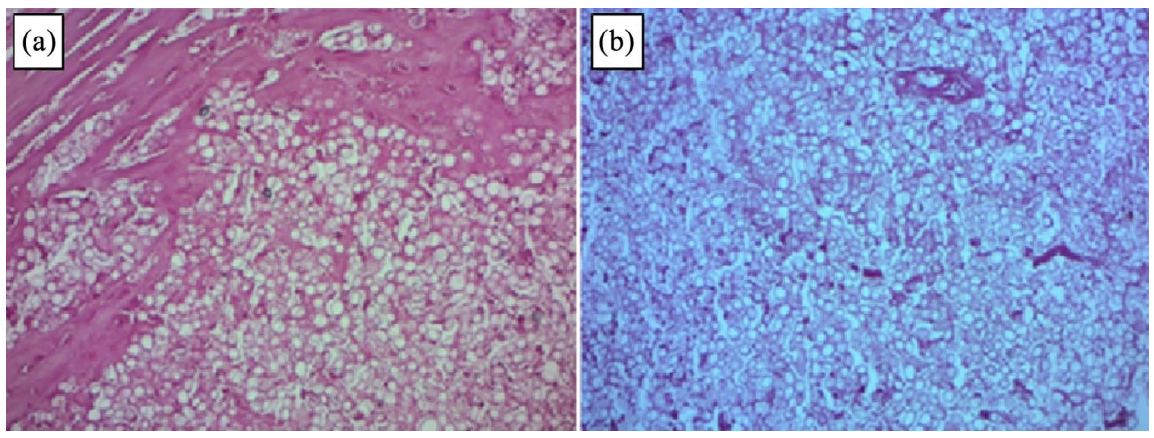

Figure 4. (a) Staining by P.A.S. (Periodic Acid Schiff) staining these forms in purple-pink, with the pseudo capsule visible. (b) Staining by P.A.S. (Periodic Acid Schiff) staining these forms in purple-pink, with the pseudo capsule visible.

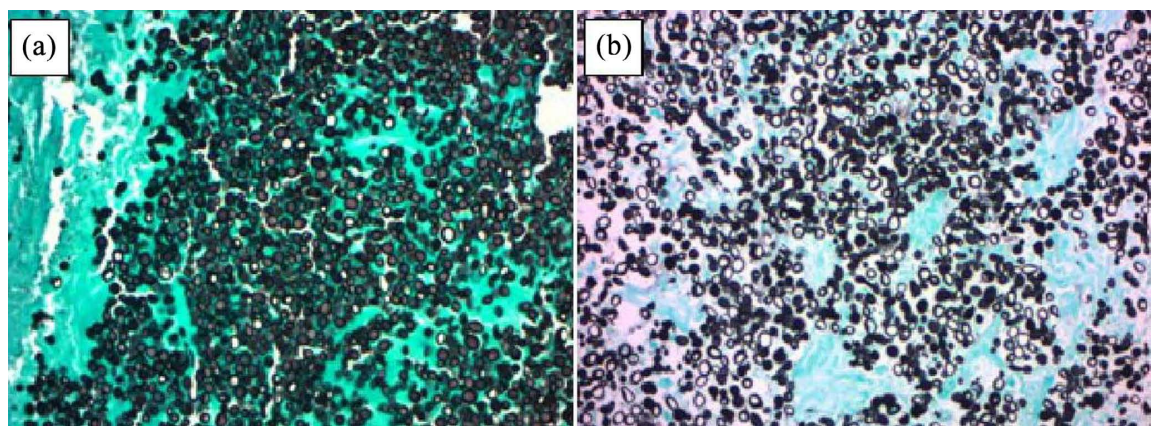

Figure 5. (a) Gomori-Grocott silver impregnation at high magnification $\times 400$, is the reference complementary stain, which shows the wall stained in black of the yeasts in intrahistiocytic position dispersed in the necrosis. (b) Gomori-Grocott silver print at high magnification $\times 400$, which shows the black-colored wall of the yeasts.

The patient was operated on for the peritoneal mass, with simple postoperative treatment followed by treatment with itraconazole at a dosage of $400 \mathrm{mg} /$ day for 14 days. The regression was favorable after 9 months of follow-up.

\section{Discussion}

African histoplasmosis is a rare granulomatous infection first described by Catanei in 1945 [4]. Caused by the dimorphic fungus Histoplasma capsulatum var. duboisii. This infection is most common in Central and West African countries. Numerous cases have been reported in Senegal [5], Mali [6], Ivory Coast [7]. The case we are reporting is original, as it is the first description of this condition in Mauritania. However, this infection has been reported in neighboring countries. Its frequency increases proportionally with $H I V$ infection [8]. Our patient was immunocompetent, with a history of pulmonary tuberculosis, which contradicts the data in the literature [8]. Histological examination of the operative specimen reveals a polymorphic granulomatous infiltrate, with multi-nucleated giant cells, within which we can distinguish multiple yeast-like bodies of large size, with thickened walls, and with a double positive contour for P.A.S and Gomori-Grocott stain characteristic of Histoplasma duboisii.

The mycological examination allows the visualization of ovoid-shaped yeasts, 
mainly intracellular. Classically, according to many authors, histopathological examination is a sensitive method that makes it possible to confirm the diagnosis in $87 \%$ of cases [9] [10]. Treatment involves itraconazole, high doses of ketoconazole can, however, give good results.

\section{Conclusion}

Histoplasmosis is a rare condition in immunocompetent patients, but not exceptional. The clinician should think about this when considering any peritoneal mass in a patient who has stayed in Central and West Africa.

\section{Conflicts of Interest}

The authors declare no conflicts of interest.

\section{Contributions from the Authors}

All the authors have marked when carrying out this work. All authors also declare that they have read and approved the final version of the manuscript.

\section{References}

[1] López-Daneri, A.G., Arechavala, A., Iovannitti, C.A., Mujica, M.T. (2016) Disseminated Histoplasmosis in Patients with AIDS. Buenos Aires, 2009-2014. Medicina, 76, 332-337.

[2] Tobón, Á.M., Medina, A., Orozco, L., Restrepo, C., Molina, D., de Bedout, C., et al. (2011) Histoplasmosis diseminada progresiva en una cohorte de pacientes coinfectados con el VIH. Acta Médica Colombiana, 36, No. 2. https://doi.org/10.36104/amc.2012.1485

[3] Ijaz, A. and Choudhury, D. (2010) A Case of Rare, Fungal Peritonitis Caused by Histoplasma capsulatum in a Patient on CAPD. Nature Reviews Nephrology, 6, 435-439. https://doi.org/10.1038/nrneph.2010.70

[4] Catanei, A. and Kevran, P. (1945) Nouvelle mycose humaine observée au Soudan Français. Archives de P Institut Pasteur d Algerie.

[5] Keita, A., Bentefouet, L. and Lawson, A.T.D. (2019) Random Diagnosis of Disseminated Histoplasmosis with Histoplasma capsulatum var. duboisii in Senegal's Hospital: Why Did One Have to Think about it? Austin Journal of Infectious Diseases, 6.

[6] Minta, D.K., Sylla, M. and Traoré, A.M., et al. (2014) Malian First Observation of Disseminated African Histoplasmosis with Predominant Bone Localizations in an HIV-Negative Child in Bamako (Mali). Journal of Medical Mycology, 24, 152-157. https://doi.org/10.1016/j.mycmed.2013.08.001

[7] Kouassi, Y.I., Ahogo, K.C. and Koui, B.B.S. (2016) Cutaneous Histoplasmosis to Histoplasma capsulatum var. duboisii: A Lethal Form in an Immunocompetent $\mathrm{Pa}$ tient. EDUCI 2016. Revue International des Sciences Médicales.

[8] Ahogo, K.C., Sangaré, A., Gbery, I.P., et al. (2009) Histoplasmose due à Histoplasma capsulatum var. duboisii à localisation cutanée chez un enfant immunocompétent. À propos d'un cas à Abidjan, Côte d'Ivoire. Bulletin de la Société de Pathologie Exotique, 102, 147-149.

[9] Maubon, D., Simon, S. and Aznar, C. (2007) Histoplasmosis Diagnosis Using a Polymerase Chain Reaction Method. Application on Human Samples in French Guia- 
na, South America. Diagnostic Microbiology and Infectious Disease, 58, 441-444. https://doi.org/10.1016/j.diagmicrobio.2007.03.008

[10] Wheat, L.J., Freifeld, A.G., Kleiman, M.B., Baddley, J.W., McKinsey, D.S., Loyd, J.E., et al. (2007) Clinical Practice Guidelines for the Management of Patients with Histoplasmosis: 2007 Update by the Infectious Diseases Society of America. Clinical Infectious Diseases, 45, 807-825. https://doi.org/10.1086/521259 